\title{
Adverse Health and Environmental Impacts of Poor Infrastructure Management in Local Market Facilities
}

\author{
Ammara Kaynat ${ }^{1}$, Ali Raza Khoso ${ }^{2 *}$, Jam Shahzaib Khan ${ }^{3}$, Rizwan Ullah Faiz ${ }^{4}$ and Mazhar Abbas ${ }^{5}$ \\ 1Department of Interior Design, National College of Arts, Lahore, Pakistan; ammarakaynat92@hotmail.com \\ 2Department of Civil Engineering, Mehran UET, Jamshoro, Pakistan; engr.aliraza23@gmail.com \\ ${ }^{3}$ Department of Civil Engineering, QUCEST, Larkana, Pakistan; jam_shahzaib@hotmail.com \\ ${ }^{4}$ School of Civil Engineering, Faculty of Engineering, Universiti Teknologi, Malaysia; rizwantn16@gmail.com \\ ${ }^{5}$ Department of Product Design, National College of Arts, Lahore, Pakistan; dr.mazhar@nca.edu.pk
}

\begin{abstract}
Objectives: This study aims to suggest infrastructure management facilities for a well-planned slaughterhouse, furthermore, proposed a systematic slaughterhouse infrastructure with modernized facilities including orientation, circulation areas, waste disposal and effluent disposal methods. Methods/Analysis: The stratified research sample was taken in the form of focused group discussions and interviews. The qualitative data thus gathered from environmentalist, civil engineers, construction managers, architects and planners to workout facility management plan for slaughterhouse. Findings: The results outlined that poor slaughterhouse infrastructure planning and management poses great threat to the human health and society by disseminating several diseases. Thereof, study concluded that the infrastructure planning can play positive and effective role to mitigate the concerned issues. Novelty/Improvement: None of the work has focused on infrastructure planning to resolve the health and environmental issues arising from the slaughterhouse's wastages. This research is novel contribution in recommending the infrastructure planning solutions.
\end{abstract}

Keywords: Health and Environment, Hyderabad, Infrastructure, Slaughter House Waste (SHW)

\section{Introduction}

Local business markets strengthen socio-economic development of the country that sustains local market facilities and opts significant assistance through infrastructure management. Arduously, amongst other local business markets the meat facilities are one of the major sources of socio-economic development. It's expanding consumption per capita and export demand makes the inevitable slaughterhouse infrastructure. They played vigorous part in meeting the meat demand, also, through livestock it contributes substantially and directly to human health. ${ }^{1}$ A better physical and psychological development is connected with this market as meat based products can reduce nutritional deficiency ${ }^{2}$. Especially, the diet deficiency in children is very common likewise other under-nourished people therefore the small amount of meat product is important for physical and psychological health.
Thus, the consequence of improper infrastructure management curtails the various physical and health problems in the demographics of slaughters houses.

The availability of infrastructure, its location and proper planning of slaughterhouses is an important and most arisen issue. This wastages contaminates the lakes, canals, rivers etc. owing to its direct flow and later become the part of human body ${ }^{1,3}$. The contaminated water coming out of slaughterhouses basically contains the fats, proteins and fibers that resulted in over contains of organic matters, thereof contaminate the rivers and drainages. The Slaughterhouse Waste (SHW) contains the solid particles; phosphorus, nitrogen etc. thus increase the $\mathrm{BOD}^{5}$ level of receiving water, potentially leading to eutrophication ${ }^{4}$. The study claims that such huge amount of SHW is a common issue of our society and a great environmental threat.

*Author for correspondence 
Slaughterhouses in Pakistan are deprived of basic infrastructure facilities for instance; wastewater drainage, water supply, ventilation, proper flooring, and transport etc. The conditions of those slaughterhouses pose severe health impacts to the society owing to unhygienic environment of the houses. The discrete disposal of waste, animal waste in hazardous manner, unhygienic air and polluted land and water, and highly polluted effluent discharge are prevailing in these houses. The research consists of an evaluation survey of infrastructure management of slaughterhouses in the vicinity of Hyderabad City, Sindh, Pakistan to highlight its major infrastructural management and planning issues. The current infrastructure of slaughterhouses is lacking the basic needs with lack of spatial requirements including the orientation (air and light) that is free from admixture with noxious elements; clean surroundings. The proper circulation spaces are utmost important for the labors to transport the animals from one place to another and drainage facilities is one of the prime need that lacks in the present houses. Mostly massive environmental perils and hygienic problem are caused because of improper initial basic amenities includes proper flooring, supply of water, transport and ventilation. Beside this, open damping of SHW are another great threat making the environment more contaminated and resulting in several chronic diseases and other public health problems, thus need a proper systematic infrastructure management and planning.

Thereof, this research focuses the rising environmental and other health issues owing to slaughterhouses and connects them with improper infrastructure facilities inside and outside the houses. More apprehension has been articulated over the hazard to the health of inhabitants of the vicinity of slaughterhouses. Many people have been expressing insecure regarding infrastructure management, location, condition and facilities management of slaughter houses that are very important $t^{4}$. The results outlined various ways for a well-planned slaughterhouse so that there should not be any bad impact of this infrastructure management on life of human being.

\section{Adverse Impact of Slaughterhouse Waste (SHW)}

Slaughterhouse Waste (SHW) constitutes the inedible parts of animals derived from the production of meat, as well as blood and other animal byproducts. The waste- water coming out of slaughterhouse poses serious health effects. Pathogens from the wastewater can be transfer to the human body once people are exposed to the water body. The water is unsuitable for drinking, swimming and even for irrigation purposes $\frac{1,3}{3}$. The pathogens proliferate to the meat because of unhygienic slaughterhouse condition. For instance ${ }^{3}$ investigated that people around the globe have experienced several chronic diseases because of slaughterhouse waste including; liver malfunctions, gastrointestinal diseases, bloody diarrhea etc. In several cases the death also occurs. Also, several cases of hepatitis A \& E viruses have been reported from the meat ${ }^{5}$. For instance ${ }^{2}$ also investigated the hepatitis viruses in slaughterhouse workers.

The effluent from the slaughterhouses is extremely hazardous and badly shakes the environment. An experimental study by 1 finds the antibiotic concentration in rivers from nursery, hospitals and slaughterhouses. The results revealed that the concentration was extremely high in the slaughterhouses effluent comparing to hospitals, making ill effects on human health. In connection with same investigation ${ }^{4}$ did an extensive experimental analysis of water quality affected by slaughterhouse of receiving rivers and concludes that the effluent is highly suspected and poses serious negative health effects on human health.

The waste water from the slaughterhouses considered damaging owing to complex mixture of fibers, fats, and proteins.

It has been considered as the most unfavorable waste water and is extremely harmful for human health, as investigated by the United States Environmental Protection Agency ${ }^{6}$. The effluent coming out of slaughterhouse contaminates the land and rivers. Therefore ${ }^{7.8}$ investigated the modern techniques to treat this waste water, else posturing ill health effects on the society. For instance ${ }^{9}$ studied the treatment, management and disposal of slaughterhouse wastewater and concludes that the water contains extremely harmful substances thus require efficient treatment.

The blood is the major dissolved pollutant of several slaughterhouse wastage. A study found that if the blood from single cow carcasses allowed penetrating the soil, it has total effluent load of 50 person per day ${ }^{10}$. Several health affects occur due to human activities inside the houses owing to poor management of effluents discharge, solid waste, and intolerable odors of the slaughterhouses therefore the proper management of slaughterhouses' waste is 
of utmost importance and proper infrastructure planning of the house seems the only solution. For instance, the installation of treatment plants. For instance ${ }^{\underline{1}}$ determined the health and environment impacts of slaughterhouse conditions on the human being while conducting a case study in India. The study found that due to poor standards of slaughterhouses the serious environmental and health hazards occurs. They further support their study by finding the health of nearby people and asserted that people are suffering with several chronic diseases. The research suggests that proper location of slaughterhouses is one of the economical solutions in this regard.

Unfolding serious concern of adverse impacts of SHW (Figure 1) the infrastructure facilities is the key solution to these problems. The most important issue is its location inside the cities. The Hyderabad, Sindh, is rapidly growing city surrounded with high demanding business markets in its hub and its surrounding thus the planning for its location is a difficult job. The residential, commercial, and economical zones of the city are highly saturated with the business activities making the city congested. Owing to these problems of Hyderabad city and looking the importance of slaughterhouses infrastructure have attracted the attention of researchers and planners. Therefore, the health and environments impacts of slaughterhouse are critically important and also discussed widely in the current literature; however, its infrastructure planning which is of worth importance has not been discussed yet. Only a study conducted by ${ }^{12}$, where the authors focus was only on its location, however, the other major

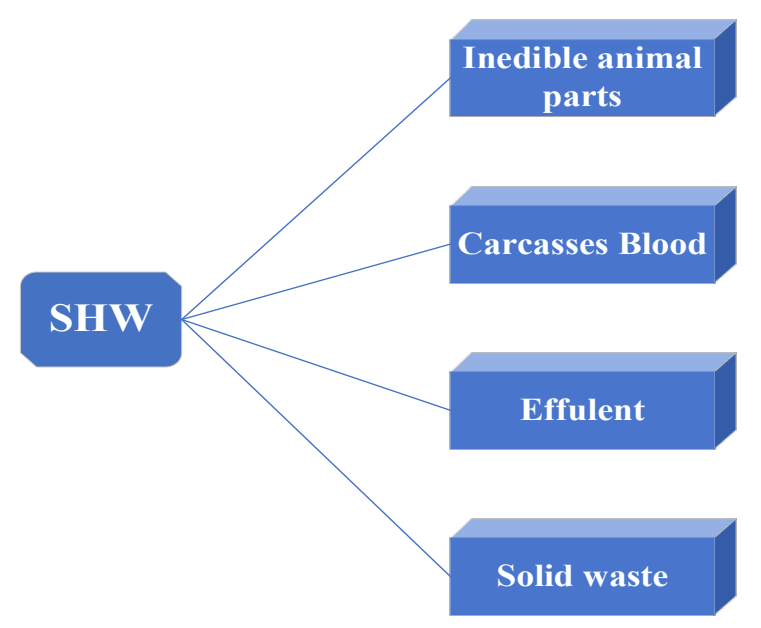

facilities for e.g., water supply and drainage facilities, circulation area, slaughterhouse inside planning, separation of slaughtering zone with other facilities, and other required amenities etc., have not been covered. This study is a novel approach to correlate the health effects arising from slaughterhouse into its infrastructure management. To best of our knowledge, none of the study has yet been carried out in connection to infra structure management of slaughterhouses, which is an important part of day to day life in any country.

\section{Research Methodology}

Meat is essential component of our daily diet; we consume meat to congregate our dietary need. The study focused geographic location of Hyderabad city, where averagely 30 to 35 animals are slaughtered every day. The lack of modern infrastructure management planning techniques is posing adverse health impacts in slaughterhouses and necessities extensive research. The literature review was unfolded to investigate the ill effects due to Slaughterhouse Wastages (SHW) in the society. A survey was carried out within different parts of Hyderabad city and its vicinity for evaluating their available infrastructure system. Stratified structured interviews were conducted with experts and professionals of various fields such as environmentalist, civil engineers, construction managers, architects and planners to impede poor infrastructure management facilities in the slaughterhouses. This determines the health issues pertaining

Figure 1. The adverse impacts of SHW.

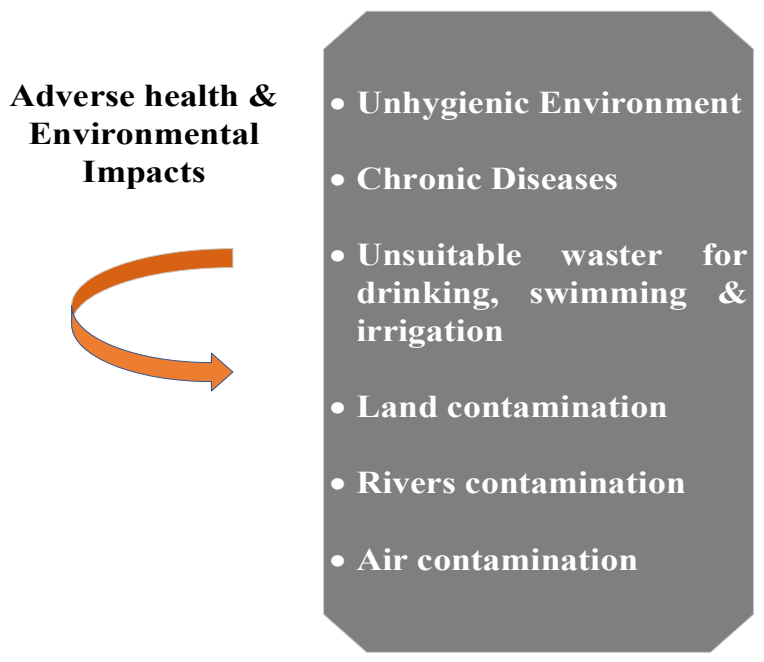




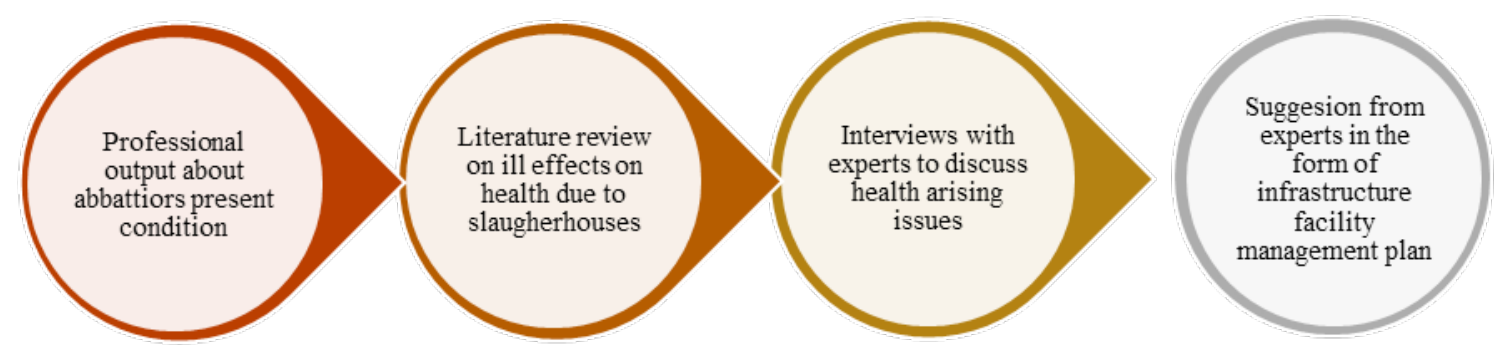

Figure 2. Conducted research methodology to achieve research aim inside facilities.

to ill planning and lack of provision of proper facilities. Later, the experts are requested to suggest the facility management plan for the slaughterhouse. Different planning strategies and solutions were gathered from different professionals to cater the health issues. Thus a suitable infrastructure facility management plan is proposed. Figure 2 illustrates the methodology conducted to achieve the research aim.

\section{Research Findings}

\subsection{Infrastructure Requirements for a Slaughterhouse}

For designing a proper slaughterhouse with all the basic amenities, a vast line of action is required for considering all those aspects which directly influence the infrastructure design and planning aspects for a slaughterhouse. These considerations include:

- Accessibility and approach from cattle market up to slaughterhouse.

- Residence animals in healthy environment.

- Appropriate circulation space for labor.

- Not physical but psychological boundaries between each process.

- Hygienic environment.

- Functional and spatial distribution.

- Accessibility from slaughterhouse up to butchers' shop, meat markets, other sources of export (bus terminals, cargo, airport).

- Orientation.

- Efficient drainage and water supply system.
The mentioned infrastructure requirements need to be properly designed and addressed before the slaughterhouse operations. The authors have proposed an infrastructure facilities management plan for a slaughterhouse. The plan can be utmost useful and effectively counter the adverse impacts associated with the operation of a slaughterhouse in a society. The architectural and engineering aspect and considerations are put together to work out the facilities.

\subsection{Infrastructure Facilities Management Plan for a Slaughterhouse}

A plan for any building or physical structure is the first representative drawing or designer's graphical representation of his technical expertise and as well as demand of public or client (depending upon nature of structure). A good plan is where functionality and economy and prime and utmost considerations. While planning for a slaughterhouse the following considerations are designed with expert's knowledge, architectural and engineering considerations and understanding. This study plans the slaughterhouse facilities into two major sub-division namely; the inside and outside facilities as illustrated in Figure 3.

\subsubsection{Healthy Residence}

A human is not safe from the cattle as numerous diseases can easily transferred from these animals to human. This problem is alarming epically for the labors working inside as causes severe symptoms leading to chronic diseases. While some of the diseases are rare, their potential for devastating outcomes makes it necessary to take precautions for these diseases seriously and residence the cattle in a healthy environment for 24 hours under the 
Inside

Facilities

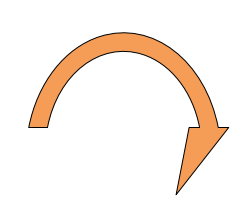

\section{Outside}

Facilities

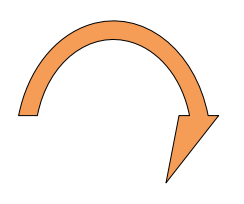

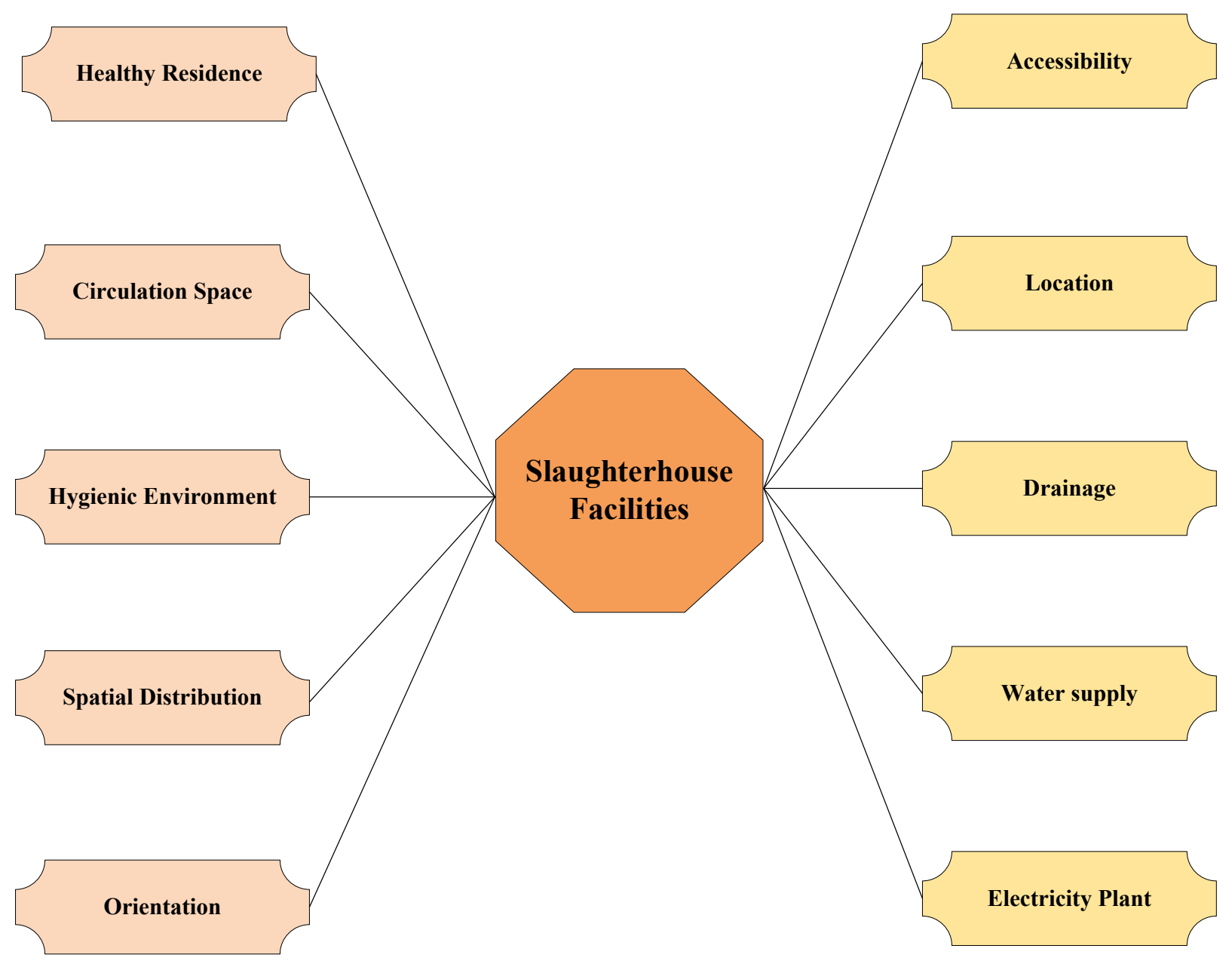

Figure 3. Inside and outside recommended facilities in slaughterhouse outside facilities.

observation of professional vet before slaughtering as the symptoms of diseases can be seen within the given time period and if that occur, the slaughtering of ill cattle will be prohibited.

\subsubsection{Circulation for Labor}

It is necessary for labor to have proper circulation; it decreases the risk of accidents and the circulation space help the people to work efficiently without the danger.
The circulation would also trigger the air entrance and circulation. The sufficient spaces inside the houses are thus necessary.

\subsubsection{Environment}

Hygienic environment in slaughterhouse is divided into two parts, one for cattle and another for slaughtering purpose. It is necessary to consider both environments because the risk of disease remains at peak before and 
after slaughtering. The air fresheners and other sprays must be installed to avoid the insects and germs to grow. Moreover, daily cleaning of cattle residence along with maintaining temperature and light is also necessary as they leave impact on cattle's health. After slaughtering the risk of virus attack increase due to raw nature of meat, here also light, air and temperature should be controlled according to the desired amount so that the meat produced under such circumstances will be hygienic.

\subsubsection{Spatial Distribution}

The slaughterhouses must be treated under special kind of planning as we consider other facilities. The design must undergo with spatial configuration which play an important role in planning slaughterhouse. Each step in the planning needs a special consideration. The sufficient spaces must be kept for the storage and functional zone.

\subsubsection{Orientation}

It refers to the direction of sunset and sunrise as well as that of prevailing wind. To obtain day light when needed and block it out when undesirable, and for ventilation throughout the building for making unwanted smell to pass out from the building. Prime factor that influence our daily routine and when talking about any functional project, there is nothing like getting ample and enough (without glare) natural light and air which help the labor to work efficiently. The buildings with proper natural light and air are energy efficient buildings. A proper lighting and air with ample number of sources is utmost important for managing proper and healthy environment inside the house. It is recommended to install larger windows with cross ventilation to catch maximum benefit from the nature. The natural light and air also useful to kill several germs and important for meat too that will also serve as self-cleaning of the houses.

\subsubsection{Accessibility}

Accessibility of slaughterhouse from cattle market is necessary for the purchasing purpose of cattle, it should be done twice or once in a week, as it is the continuous process so it must be near cattle market so that the administration can purchase the cattle easily. For this reason, the slaughterhouses are encouraged to located far away from the main business area of the city, this would further avoid the chances of environmental deterioration.

\subsubsection{Location}

Slaughterhouse must not be very near the residential areas in a city. Their presence inside the main city deteriorates the environment and increases the SHW on the streets and main roads. It is always recommended to locate them outside populated areas downwind from the city far away from water body so that the water should not be contaminated from carcasses, blood or other infected materials. This would further reduce the chances to not degrade the environment and not to harm the health of the residents.

\subsubsection{Drainage}

Drainage is one of the most important parts of slaughterhouse, as the complete process is full of blood, water and other draining stuff so the drainage must be of desired size so that it can hold all the wastage. Since, the blood of slaughtering animal when drains inside the land, and water contaminate the environment, thus a separate drainage facility is required to be designed for blood only. A separate drainage must be designed for the water; during slaughtering the animal and washing the meat etc. so that the meat intake water must be gathered separately and must not mixed with blood and other normal water. The accumulated water must be stored in septic tank and must be treated before discharging it into rivers. The blood must be accumulated separately, later; the blood can be collected and may be used for several purposes such as for medicines production.

\subsubsection{Water Supply System}

Slaughtering process continuously requires water for making the meat healthy and making the environment hygienic. In order to keep the meat hygienic, drinking water must be installed in slaughterhouse otherwise it would deteriorate the meat quality. Furthermore, to make it more efficient, wastewater treatment plant should be installing in order to recycle the water to some extent. The partially treated water can be used during slaughtering and can be used for cleaning of cattle's and their residence.

\subsubsection{Electricity Plant}

Slaughterhouse is an industrial project and affects the country economy as well. It further full fills the demand of several people. The slaughterhouse contains heavy 
machinery and chiller systems for several functions. To make slaughterhouse an efficient and healthy the electricity is required for its several functions including safe cutting, maintaining the quality by storing the meat into refrigerators etc. Electricity is also required for water and light purpose. For a bid slaughterhouse special care is needed for electricity. Additionally, the power backup system such as generator, and solar panels are also required to produce the health meat and to meta the demand.

\section{Conclusion and Future Directions}

Local business such as meat production significantly important for society from economic and human diet perspective. But several studies argued that the human health and environment negatively affected due to the ill infrastructure planning of the structures where animals are slaughtered. The literature was reviewed to investigate the consequences of the Slaughterhouses Wastages (SHW) in the society. Moreover, a small-scale survey was conducted to receive the meat user's and other people living surrounding the houses. The study found that the slaughterhouses pose great threat to the human health and to the society by propagating several diseases via. contaminating the land, air and water. Several interviews were conducted from environmentalist, civil engineers, construction managers, architects and planners in order to point out the slaughterhouse planning problem and their necessary measures. The study concludes that the infrastructure planning can play positive and effective role to mitigate the concerned issues.

This research proposed slaughterhouse infrastructure facilities management plan with all the modern facilities; inside and outside the house. The research findings are provided in the form of several recommendations to deal with the infrastructure planning solution in the form of infrastructure management plan. The study focuses the infrastructure elements of slaughterhouses from the experts view-point which is eye-opening and a premier study in this research area. However, a detailed examination can be conducted further to workout effective design solutions.

\section{Research Implication}

The study findings have several directions from applied research and practical side. The study is an eye-opening for environmentalist, decision and policy makers, and
Govt. entities. Being the premier study in the realm, this work opens several new challenges and their detailed examination for researchers. Thus, the most ignored research area would attract the researchers and other stakeholders around the globe.

The study has implication especially for the public residing surrounding the slaughterhouses, the major stakeholders of this meat production business in the form of counter measures they need to take. The environmentalist, engineers, architects and planner would deeply examine the issues further.

\section{References}

1. Chang X, Meyer MT, Liu X, Zhao Q, Chen H, Chen J. Determination of antibiotics in sewage from hospitals, nursery and slaughter house, wastewater treatment plant and source water in Chongqing region of Three Gorge Reservoir in China. Environmental Pollution. 2010; 158(5):1444-50. https://doi.org/10.1016/j.envpol.2009.12.034 PMid:20096493

2. Tariq H, Kamal MU, Makker J, Azam S, Pirzada UA, Mehak V. Hepatitis in slaughterhouse workers. World Journal of Gastroenterology. 2019; 11(1):37-49. https://doi.org/10.4254/ wjh.v11.i1.37 PMid:30705717 PMCid:PMC6354121

3. Barrera M, Mehrvar M, Gilbride KA, McCarthy LH, Laursen AE, Bostan V. Photolytic treatment of organic constituents and bacterial pathogens in secondary effluent of synthetic slaughterhouse wastewater. Chemical Engineering Research and Design. 2012; 90(9):1335-50. https://doi.org/10.1016/j.cherd.2011.11.018

4. Ayenew T, Hailu AM, Mulu A, Berhe S. Impact of slaughterhouses effluent on water quality of Modjo and Akaki river in Central Ethiopia. International Journal of Science and Research. 2015; 4(3):899-907.

5. PinaS,ButiM,CotrinaM,PiellaJ,GironesR.HEVidentifiedin serum from human with acute hepatitis and sewage of animal origin in Spain. Journal of Hepatology. 2000; 33(5):826-33. https://doi.org/10.1016/S0168-8278(00)80316-5

6. Effluent limitations guidelines and new source performance standards for the meat and poultry products point source category. Available from: https://www. federalregister.gov/documents/2004/09/08/04-12017/ effluent-limitations-guidelines-and-new-source-performance-standards-for-the-meat-and-poultry

7. Bustillo-Lecompte CF, Mehrvar M, Quiñones-Bolaños E. Cost-effectiveness analysis of TOC removal from slaughterhouse wastewater using combined anaerobicaerobic and $\mathrm{UV} / \mathrm{H}_{2} \mathrm{O}_{2}$ processes. Journal of Environmental Management. 2014; 134:145-52. https://doi.org/10.1016/j. jenvman.2013.12.035 PMid:24486468 
8. Kundu P, Debsarkar A, Mukherjee S, Kumar S. Artificial neural network modelling in biological removal of organic carbon and nitrogen for the treatment of slaughterhouse wastewater in a batch reactor. Environmental Technology. 2014; 35(10):1296-305. https://doi.org/10.1080/09593330.2 013.866698 PMid:24701927

9. Ciro, Mehrab MB-L. Slaughterhouse wastewater: treatment, management and resource recovery. In: Physico-Chemical Wastewater Treatment and Resource Recovery; 2017. p. $153-70$.
10. Aniebo AO, Wekhe SN, Okoli IC. Abattoir blood waste generation in rivers state and its environmental implications in the Niger Delta. Toxicological and Environmental Chemistry. 2009; 91(4):619-25. https://doi.org/10.1080/02772240802343404

11. Singh AL, Jamal S, Baba SA, Islam MM. Environmental and health impacts from slaughter houses located on the city outskirts: A case study. Journal of Environmental Protection. 2014; 5(6):566-75. https://doi.org/10.4236/jep.2014.56058 